\title{
In Search of the Chimera: Molecular Imaging in the Atom-Probe
}

\author{
J. A. Panitz* \\ *High-Field Consultants, Inc. Sandia Park, NM 87047.
}

The Atom-Probe Field Ion Microscope is the ultimate microanalytical tool because a single atom can be chosen from its neighbors at the discretion of the observer and identified in a time-of-flight mass spectrometer [1]. A simplification of the apparatus called the 10-cm Atom-Probe (Fig. 1) extended the analysis capability of the original instrument [2]. With the introduction of the Imaging Atom-Probe a preselected species could be visualized in atomic resolution and mapped in three-dimensions with a lateral and depth resolution exceeding $0.5 \mathrm{~nm}$ under ideal conditions [3]. Although Atom-Probe analysis has been confined to surface studies and problems in the materials sciences the lure of imaging and analyzing individual molecules has been strong. See a review of attempts to image individual molecules in the Field-Emission Electron Microscope and the Field-Ion Microscope for details [4].

\section{Sample Preparation}

If a molecule, such as copper phthalocyanine, can be sublimed onto a substrate in high vacuum sample preparation presents no difficulty. Unfortunately, most molecules of interest (DNA, proteins or virus particles) must be deposited onto a substrate from aqueous solution and then transferred into high vacuum for imaging. Early attempts to image these molecules were hampered by an inability to independently verify the success of the deposition process. This obstacle was overcome by using the Transmission Electron Microscope (TEM) to verify protein deposition on field-emitter tips (Fig. 2) [5]. A refinement of the deposition protocol minimized the drying artifacts associated with surface tension forces acting on the sample as it is moved through an aqueous interface and dried in air [6]. For a molecule of characteristic dimension, $\mathrm{d}$, in meters surface tension results in a pressure, $\mathrm{Pg} \approx 0.146 / \mathrm{d} \mathrm{N} / \mathrm{m}^{2}$ that can redistribute molecules on the surface and distort or destroy their tertiary structure [7].

\section{Imaging Constraints}

The electric field strength generated at the substrate is the greatest obstacle to successful imaging of molecules in the Atom-Probe. The magnitude of the field, E, that can redistribute molecules on the surface and distort or destroy their tertiary structure can be estimated by assuming the outward-directed electrostatic pressure is equivalent to $\mathrm{Pg}$. Then $\mathrm{E} \approx\left(2 \mathrm{Pg} / \varepsilon_{0}\right)^{1 / 2}=\left(0.292 / \varepsilon_{0} \mathrm{~d}\right)^{1 / 2} \approx 18 \mathrm{MV} / \mathrm{cm}$ which is at least an order of magnitude below the field strength required for imaging in hydrogen or helium.

\section{Field Ion Tomography}

Stable and reproducible images of a ferritin monolayer on a tungsten surface were obtained in the Imaging Atom-Probe by embedding ferritin within a layer of vitreous benzene ice condensed onto an $80 \mathrm{~K}$ surface using gas phase benzene as a blanket gas [4]. As the field is increased, benzene is desorbed from the surface as cluster ions $\left.\left(\mathrm{C}_{6} \mathrm{H}_{6}\right)_{\mathrm{n}}{ }^{+}, \mathrm{n}=1,2\right)$ which expose the contour of ferritin molecules as a function of depth within the benzene layer (Fig. 3). This process, called Field-Ion Tomography can be used to reconstruct the three-dimensional morphology of a ferritin monolayer (Fig. 4) [8]. Image resolution is limited by the size of the benzene cluster ions to $\approx 2 \mathrm{~nm}$ and the field strength for benzene desorption $(\approx 4 \mathrm{MV} / \mathrm{cm})$ is well below the field required to distort, destroy, or desorb the ferritin monolayer from the surface [8]. Cryofixation in vitreous water ice and cryotransfer into the Atom-Probe has been demonstrated and could improve the resolution of the imaging process [9]. 


\section{References}

[1] E. W. Muller, J. A. Panitz and S. B. McLane, Rev. Sci. Instrum. 39 (1968) 83.

[2] J. A. Panitz, Rev. Sci. Instrum. 44 (1973) 1034.

[3] J. A. Panitz, J. Vac. Sci. Technol. 11 (1974) 206.

[4] J. A. Panitz, J. Microsc. 125 (1982) 3.

[5] J. A. Panitz and I. Giaever, Surface Sci. 97 (1980) 25; Ultramicroscopy 6 (1981) 3.

[6] J. A. Panitz, Rev. Sci. Instrum. 56 (1985) 572.

[7] A. A. Bartlett and H. P. Burstyn, Scanning Electron Microsc. 75 (1975) 305.

[8] J. A. Panitz, Ultramicroscopy. 7 (1982) 241.

[9] J. A. Panitz and A. Stintz, Surface Sci. 246 (1991)163; J. Appl. Phys. 72 (1992) 741.

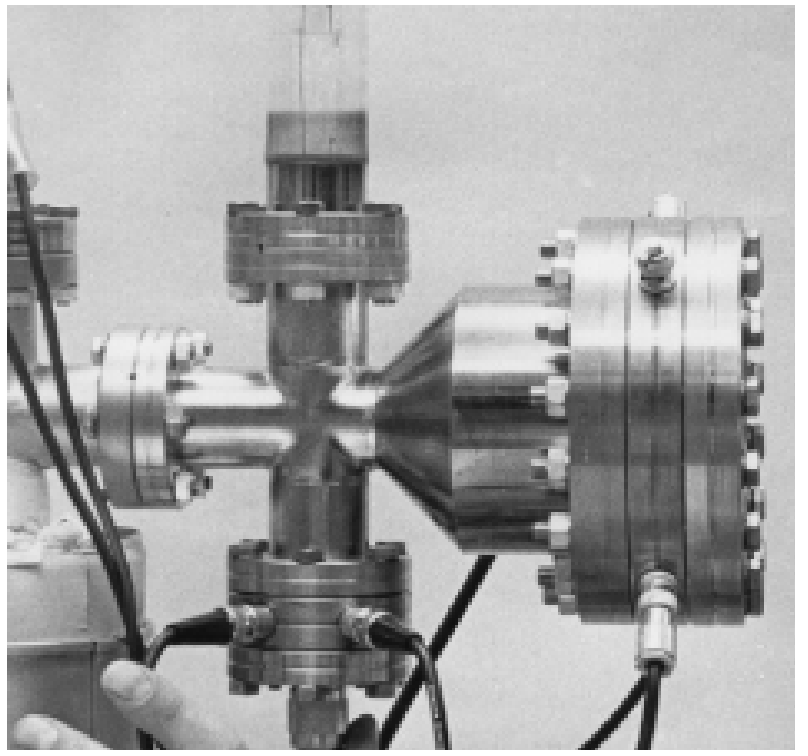

Fig. 1. The 10-cm Atom-Probe.

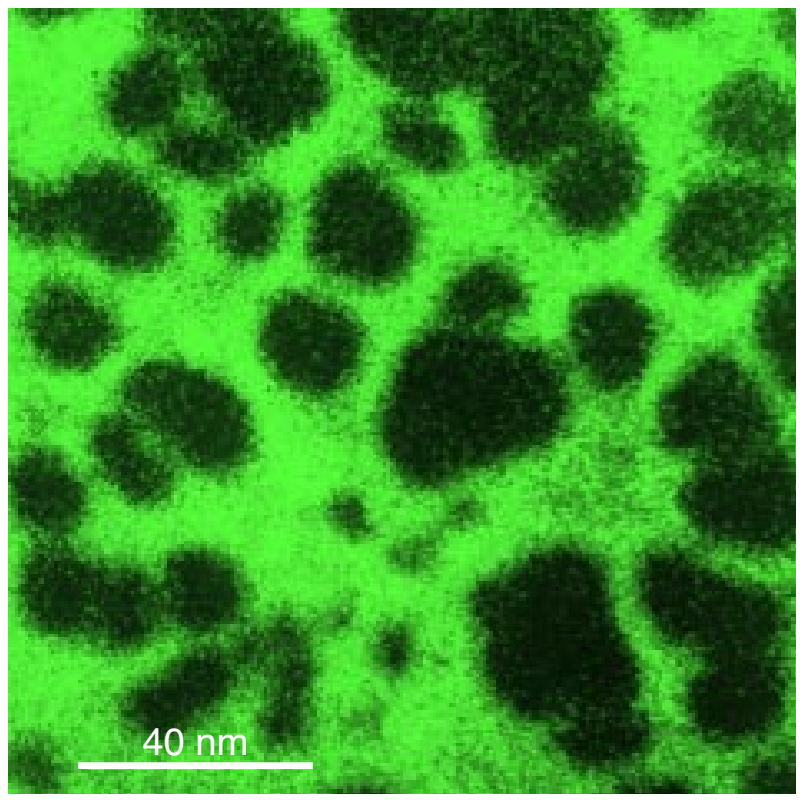

Fig.3. Field-Ion Tomography of Ferritin.

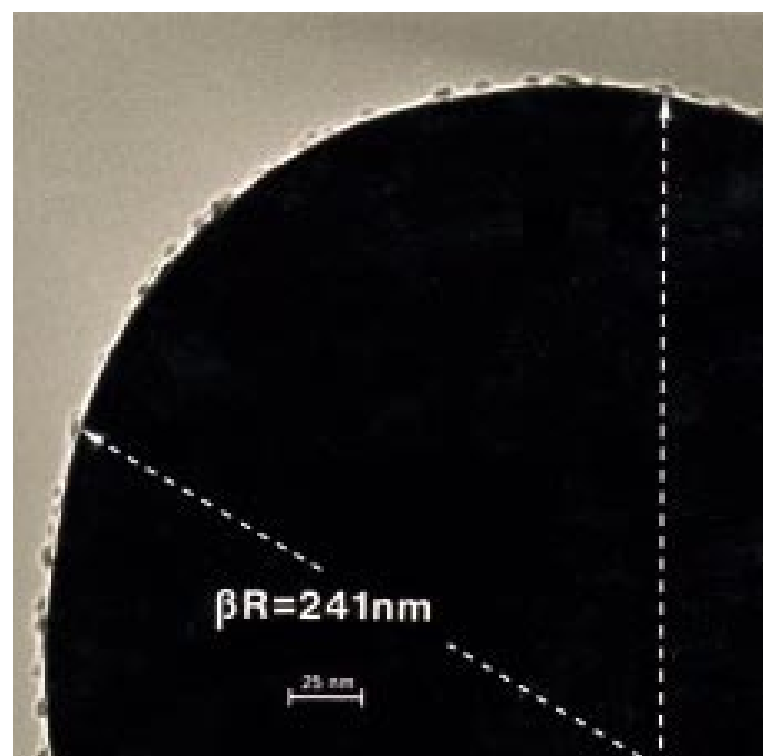

Fig. 2. A Ferritin Monolayer on Tungsten ( $\beta \approx 1.5)$.

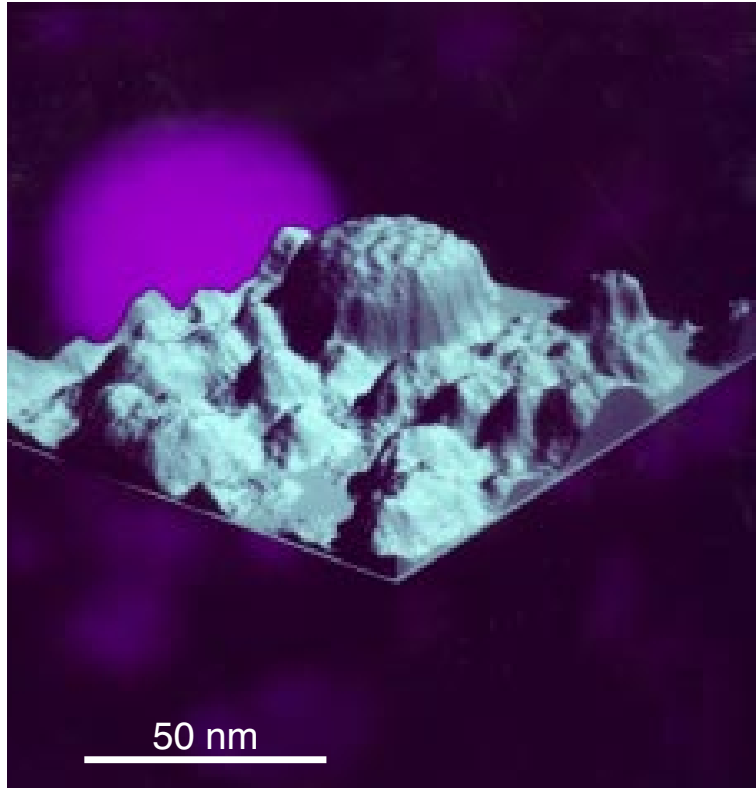

Fig. 4. A Tomgraphic Reconstruction of Ferritin. 